\title{
Procedimientos de control interno para el ciclo de ventas, cuentas por cobrar y cobros
}

Internal control procedures for the sales cycle, accounts receivable and collections

\author{
Génesis Lara \\ genelara@hotmail.com \\ ORCID: 0000-0003-3246-3299
}

Universidad de Carabobo, Valencia - Venezuela
Lorna Pérez

daniela13594@hotmail.com

ORCID: 0000-0002-1255-3425

Universidad de Carabobo, Valencia - Venezuela

Artículo recibido febrero 2020 / Arbitrado en marzo 2020 / Aceptado junio 2020 / Publicado en julio 2020

\section{RESUMEN}

La presente investigación tiene como objetivo proponer procedimientos de control interno para el ciclo de ventas, cuentas por cobrar y cobros en la empresa Floristería Bárbara, C.A. Metodológicamente la investigación se categoriza como un estudio de campo no experimental, bajo la modalidad de proyecto factible. El estudio fue complementado con fuentes bibliográficas-documentales, observación directa. La población fue de cinco (5) personas del Departamento de Administración y Finanzas. Para la recolección de datos se aplicó una lista de cotejo, una encuesta. Los resultados demostraron que, en los objetivos organizacionales y financieros del ciclo de ventas, cuentas por cobrar y cobros de la empresa no están definidos y no son conocidos por el personal, situación respaldada por el 80 \% consultado. Es relevante el empleo de controles para el área de ventas, cuentas por cobrar y cobros, es fundamental que exista un ambiente de control y seguimiento eficiente en las actividades operacionales.

Palabras Claves: Control interno, eficacia y eficiencia operativa, proceso de cuentas por cobrar, plan de gestión administrativa, ventas

\begin{abstract}
The purpose of this research is to propose internal control procedures for the sales cycle, accounts receivable and collections at the company Floristería Bárbara, C.A. Methodologically, the research is categorized as a non-experimental field study, under the feasible project modality. The study was complemented with bibliographicdocumentary sources, direct observation. The population was five (5) people from the Administration and Finance Department. For data collection a checklist, a survey, was applied. The results showed that in the organizational and financial objectives of the sales cycle, accounts receivable and collections of the company are not defined and are not known by the personnel, a situation supported by $80 \%$ consulted. The use of controls for the sales area, accounts receivable and collections is relevant; it is essential that there be an efficient control and monitoring environment in operational activities.
\end{abstract}

Key Words: Internal control, effectiveness and operational efficiency, Accounts receivable process, administrative management plan, sales 


\section{INTRODUCCIÓN}

El flujo de efectivo es uno de los recursos indispensables que requiere cualquier organización para su funcionamiento eficiente, pues a través del mismo se puede lograr la compra de bienes, productos y servicios cuyos proveedores no siempre aceptan formas diferidas de pago, tal como ocurre con las mercancías para la venta, y realización de compras e inversiones de diverso tenor, en el corto y mediano plazo. Es por ello, que su correcta administración es crucial, para garantizar su operatividad y continuidad dentro de su mercado de referencia.

De manera que, esta ha sido la gran preocupación de muchos directores y gerentes, que el efectivo que ingresa a la organización tenga un flujo constante y sea bien utilizado, para que se alcancen los fines propuestos y establecidos por los propietarios y accionistas. En consecuencia, la empresa debe procurar mantener la disponibilidad del efectivo presupuestado para que sus costos y gastos sean cubiertos en el momento oportuno.

Es por este motivo, que en los casos de las empresas que venden a crédito, deben tener unos criterios cónsonos con la situación económica del lugar en la cual están establecidas. Las cuentas por cobrar representan un instrumento realmente importante en la función financiera de una empresa. De una buena política de concesión de crédito y de un buen sistema de cobros, dependerá la contribución de las cuentas por cobrar a la provisión de fondos en las condiciones más ventajosas y a la maximización de las utilidades de la empresa. Es relevante señalar, lo que expone Rosero (2014) quien argumenta sobre la importancia de los controles internos en cuentas por cobrar de la siguiente manera:
El Control Interno surge desde la necesidad de evaluar el proceso de la Administración, en controlar las actividades pues es fundamental para el logro de objetivos propuestos, por ende es la base para que la Empresa alcance en forma ascendente los procesos y procedimientos productivos para la toma de decisiones (p.26)

Por otra parte, para desarrollar un procedimiento de control interno es imprescindible conocer las características por consiguiente se debe considerar que el control interno, lo constituyen los planes, métodos, técnicas y procedimientos que sigue una entidad de acuerdo a sus políticas contables, para proteger su activos, contra erogaciones impropias de sus activos, para prevenir la ocurrencia de obligaciones inadecuadas, con la intención de que los procedimientos contables y administrativos originen información financiera razonable y confiable.

En este sentido, el control se puede considerar como una etapa primordial del proceso administrativo, pues, aunque una empresa cuente con unos planes, una estructura organizacional adecuada y una dirección eficiente, el ejecutivo no podrá verificar cuál es la situación real de la organización si no existe un mecanismo que se cerciore e informe si los hechos van de acuerdo con los objetivos.

Esto demuestra, que los controles internos, tienen la finalidad de proteger y resguardar los activos de la entidad y evitar pérdidas financieras futuras, que en este caso particular, estarían representadas por las facturas de la mercancía vendida a crédito, las cuales deben retornar en efectivo, dentro de su ciclo normal de operaciones, para que se garantice la 
operatividad y rentabilidad de sus procesos financieros. Si se presentan retrasos en los cobros, la empresa estaría entrando en momento de iliquidez financiera, trayendo como consecuencia, el estancamiento y las pérdidas en sus inventarios, es por esta razón, que los controles internos no deben dejarse de aplicar de manera correcta.

Es importante, para cualquier organización contar con eficientes controles internos que constituyan una guía para el proceso mediante el cual las operaciones se optimicen, pues hoy día en las empresas, independientemente de su dimensión, es necesario que cuenten con un sistema de control de los procesos contables, ya que siempre existirá la necesidad de manejar un sistema de control, con el propósito de lograr objetivos con la mayor eficiencia posible y de esta forma crecer dentro de su actividad.

En el mismo sentido, Marín (2014:2) expone de manera explicativa el modelo COSO, según dicho autor "pretende que los interlocutores tengan una referencia conceptual común sobre el control interno para garantizar un buen gobierno corporativo", por lo que el control interno cuenta con una estructura, que de ella se forman las normas y procedimientos administrativos y contables.

Es conveniente reiterar, que el sistema de Control Interno forma parte de las actividades operacionales de la entidad, no agregado a ellas, es un instrumento para alcanzar un fin, no un fin en sí mismo, un medio para lograr propósitos de eficiencia y eficacia. También es parte de los procesos que concretan la misión de la entidad y, como un sistema, se extiende por todas las actividades de la organización. Sus cinco componentes están integrados al proceso de gestión y representan lo que se requiere para cumplir cabalmente los objetivos de la entidad. Una definición condensada de estos cinco componentes, a partir de lo relacionado por Pablos, Ramírez y Román.

Es por ello, que las principales consideraciones que se deben tener para el control de las cuentas por cobrar comprende: el control independiente de los registros de dichas cuentas, generación continua de reportes que señalen los saldos deudores corrientes con su correspondiente análisis de detalles, y por último, el envío de los estados de cuenta de los clientes en forma directa e independiente.

De esta manera, las medidas de procedimientos control interno enfocado al manejo del ciclo de las cuentas por cobrar y cobros, promueven a la eficiencia, reducen riesgos de pérdidas de activos, y ayudan a asegurar la confiabilidad de los estados financieros y el cumplimiento de las leyes y regulaciones, pues el control facilita la delegación de funciones y el trabajo en equipo, además agrega valor al trabajo porque los hace participativo y evalúa áreas claves del departamento de cuentas por cobrar.

En este sentido, los sistemas de control contienen registros que permiten controlar $y$ evaluar acciones de los empleados en función al buen funcionamiento del sistema de control de procesos contables, y de esta manera, garantizarle la eficiencia y efectividad en el área contable.

Dentro de lo planteado, se presenta el caso de la empresa Floristería Bárbara, C.A., la cual es una PYME dedicada a venta y distribución de flores al mayor y al detal en el Estado Carabobo, esta organización ha presentado un crecimiento sostenible, sin embargo, producto de la falta de experiencia, así como la ausencia 
de capacidades técnicas ha llevado al empresario a no fijar correctamente los objetivos, a desconocer las realidades del entorno y posibilidades reales de la organización dentro de su ámbito de acción.

En este sentido, los procesos operativos y contables relacionados con el ciclo ventas, cuentas por cobrar y cobros que se lleven a cabo en la empresa deben proporcionar información precisa, manejable y de fácil interpretación, que además refleje la realidad de la empresa coherente y fiable que sirva de soporte a la toma de decisiones.

Sin embargo, de acuerdo con información proporcionada por la gerencia de la empresa, la organización aplica la política de otorgar créditos a sus clientes, con la finalidad de incrementar las ventas, conservar los clientes actuales y atraer nuevos, pero dichos créditos no han sido cancelados a la fecha de su vencimiento, lo que ha ocasionado problemas en el sistema de cobranza. Se observa, que existen facturas cobradas que no se han rebajado de las cuentas por cobrar y facturas que son rebajadas sin orden cronológico, debido a que el sistema actual de trabajo es manejado manualmente, lo cual ocasiona una serie de errores administrativos y contables.

Adicionalmente, en esta empresa no existe provisión para cuentas dudosas, ya que no poseen el respaldo económico necesario para solventar a corto plazo la falta de liquidez, y evidentemente el problema de cartera surge principalmente cuando la empresa ofrece crédito a sus clientes sin el análisis adecuado de sus políticas de crédito y/o de la falta de control y vigilancia de la cartera.

En lo que respecta al efecto de la falta de control interno sobre la cuentas por cobrar a clientes, se ve afectado el flujo del efectivo de la empresa, por lo que esta ausencia de control contribuye a limitar las operaciones normales de la empresa, reduce la capacidad de pagar puntualmente a los proveedores de la entidad y restringe las posibilidades de inversiones, como también, de cierto modo, limita recibir financiamiento de terceros, es decir, que no disponer del efectivo, reduce las oportunidades de crecimiento futuro de la entidad.

Asimismo, la falta de controles ocasiona que los procesos contables y administrativos se lleven de manera errónea, lo que puede afectar resultados contables de un período determinado, además influye en la inadecuada toma de decisiones. Es por esto que la empresa requiere de procedimientos que permita controlar y comprobar sus cuentas por cobrar.

Razón por la cual, esta investigación tuvo como objetivo proponer procedimientos de control interno para el ciclo de ventas, cuentas por cobrar y cobros en la empresa "Floristería Bárbara, C.A. que permitan mejorar la rentabilidad de la empresa.

\section{MATERIALES Y MÉTODOS}

La investigación se categoriza como un estudio de campo no experimental, bajo la modalidad de proyecto factible. El estudio fue complementado con fuentes bibliográficasdocumentales, observación directa.

La población fue de cinco (5) personas del Departamento de Administración y Finanzas. En vista que la población está conformada por un número finito y accesible de elementos, ésta se escogerá completa, entre el personal gerencial y empleados, por lo cual no se aplicarán técnicas muéstrales.

En cuanto a las técnicas e instrumentos de recolección de datos aplicadas con el fin de obtener la información necesaria para el 
desarrollo de la investigación, se empleará una lista de cotejo y una encuesta.

\section{RESULTADOS Y DISCUSIÓN}

Una vez aplicado los instrumentos se pudo evidenciar que el $80 \%$ de la población coincide que la empresa no cuenta con un perfil organizacional, se evidencia la existencia de políticas contables y su comunicación al personal, pero no se ha delimitado formalmente las responsabilidades de cada integrante de la empresa, así como también que no existen normas y procedimientos para cada uno de los procesos de la empresa, con respecto a la aplicación de métodos de control interno en la gestión de cuentas por cobrar de la empresa.

Además, se comprobó que el control administrativo para la gestión de ventas, cuentas por cobrar y cobranzas debe surgir en la empresa como una herramienta de trabajo, con la intención de verificar que las operaciones se realicen de acuerdo a las autorizaciones generales y específicas impuestas por la administración; que todas las operaciones se registren oportunamente y por los montos correctos, en las cuentas apropiadas y en el periodo contable en el cual se llevan a cabo; que el acceso a los activos de la empresa sea solamente con autorización administrativa, y que todo lo contabilizado exista, realizando investigaciones para detectar cualquier diferencia y aplicar los correctivos necesarios.

En este sentido, la importancia de las cuentas por cobrar es inmensa e indispensable para la gestión económica eficaz, al resguardar asertivamente el flujo oportuno de los recursos financieros requeridos para la producción y rentabilidad organizacional, tomando en cuenta las políticas de crédito. Este aspecto, es el que indica bajo qué circunstancias se va a conceder el crédito, es decir, los requisitos que se le solicite al cliente, así como también, el valor mínimo o máximo del menciona do. Para sintetizar, ésta aclara los parámetros para la atención de los clientes. Como afirma Catacora (2013):

Los estándares de créditos definen los criterios mínimos para la concesión de crédito al cliente, como por ejemplo las clasificaciones de crédito, referencias, plazos medios de pagos índices financieros, etc. Las políticas de crédito dependen de ciertas variables que pueden reducir o ampliar los estándares (p. 62).

En términos generales, Méndez (2016:66) indica que "las políticas de crédito y cobranza forman las bases para evitar pérdidas por concepto de entregar mercancías sin evaluar el estudio del perfil del cliente". El autor indica además que las experiencias contables de muchas empresas en este aspecto, indican que después de otorgan mercancía a crédito a sus clientes, les cuenta recuperar costos sobre la inversión debido a la baja capacidad financiera de cliente para asumir compromisos.

Por todo lo señalado anteriormente y por el interés de los miembros del departamento, en participar en un proyecto con miras al mejoramiento de los procesos y la procura de un crecimiento continuo, se realiza la siguiente propuesta 
PROCEDIMIENTOS QUE CONTRIBUYAN CON LA EFICIENCIA DEL CONTROL INTERNO EN EL CICLO DE VENTAS, CUENTAS POR $\begin{array}{llll}\text { COBRAR } Y \text { COBROS EN LA } & Y \text { N }\end{array}$ EMPRESA"FLORISTERÍA BÁRBARA, C. A."

La cual pretende mostrar una serie de controles y estrategias para el adecuado manejo del ciclo de ingresos (ventas, cuentas por cobrar y cobros) para la empresa "Floristería Bárbara". La finalidad es proponer una guía para el control y la supervisión de las actividades, de modo tal que se pueda cuantificar y calificar los resultados de las operaciones y del personal involucrado en los procesos, con la implementación de lineamientos derivados de objetivos estratégicos y objetivos tácticos. Dichas actividades comprenden las áreas de políticas de cobranzas, organización del departamento, y controles para incrementar la eficiencia del departamento de Administración, responsable del ciclo objeto de estudio.

\section{Desarrollo de la propuesta}

Fase 1: Definición de normativas de control interno para evaluar el desempeño del personal encargado de efectuar los procesos de cuentas por cobrar y cobranza.

Esta parte de la propuesta se hace para incrementar la razonabilidad y coherencia en la presentación de los estados financieros de la empresa y el control de operación del negocio o en las funciones implicadas.

Fase 2. Definición de las políticas adecuadas para la gestión del ciclo de ingresos de la empresa, basada en criterios de eficiencia, estandarización y transparencia.

El departamento de Administración debe establecer políticas por escrito por las cuales regirse al momento de tratar con los clientes.
Fase 3. Cumplimiento de las metas organizacionales establecidas por la empresa, a través de la articulación de todo el personal que integra la gestión del ciclo de ingresos.

Fase 4: Definición de indicadores de gestión para medir la eficiencia en la gestión del ciclo de ingresos y contribuir a la toma de decisiones oportunas.

Esta fase consistirá en la selección del modelo estratégico para el diseño y presentación del sistema de indicadores de gestión y luego la selección de los indicadores:

- Análisis de las bases de datos y documentos disponibles para crear los indicadores de gestión.

- Diagnosticar la situación de las estrategias propuestas.

- Determinar los puntos de inflexión y elementos susceptibles de ser medidos.

- Formalización del sistema de indicadores.

- Selección de los indicadores de gestión adecuados, en atención a los mencionados anteriormente.

\section{CONCLUSIÓN}

A lo largo de la realización del presente estudio, se observó la imperante necesidad por parte del Departamento de Administración de la empresa "Floristería Bárbara", C.A., de contar con estrategias acordes con la naturaleza del negocio y las necesidades del mercado al cual atiende; y que además, se someta a evaluaciones constantes de su funcionamiento administrativo, con la finalidad de que la empresa se adapte al medio ambiente que se encuentra en un continuo proceso de cambio.

Debido a lo anteriormente expuesto, estos análisis se deben realizar en cualquier tipo de organizaciones, sean públicas o privadas, o se 
dediquen a la venta de bienes o la prestación de servicios; en fin, a toda empresa, independientemente de la actividad $u$ objetivo que persiga.

Una vez analizado todos los resultados obtenidos se precede hacer las siguientes recomendaciones, verificar que las operaciones se realicen de acuerdo a las autorizaciones generales y específicas impuestas por la administración; que todas las operaciones se registren oportunamente y por los montos correctos, en las cuentas apropiadas y en el periodo contable en el cual se llevan a cabo; que el acceso a los activos de la empresa sea solamente con autorización administrativa, y que todo lo contabilizado exista, realizando investigaciones para detectar cualquier diferencia y aplicar los correctivos necesarios.

En cuanto a la revisión y divulgación de los manuales sobre normas y procedimientos, es necesario que permitan la elaboración y/o actualización de los manuales sobre normas y procedimientos relacionados en el área administrativa y ciclo de cuentas por cobrar, orientados en una forma sencilla $y$ esquematizada que permita a los trabajadores la rápida comprensión de los procesos administrativos y contables, así mismo, se requiere de la actualización de estos manuales por parte de las autoridades responsables para generar la información en la oportunidad debida para no debilitar el proceso.

Por otra parte, es importante suministrar adiestramiento al personal para incrementar la calidad y el valor del capital humano, el cual se debe iniciar haciendo un levantamiento de información del perfil profesional del personal que labora en las áreas administrativas, número de horas de adiestramiento recibido, tópicos de adiestramiento recibido, tiempo en la organización y en el cargo, edad, sexo. Una vez procesada la información anterior, se debe elaborar un programa de adiestramiento que atienda al conocimiento necesario de las cuentas por cobrar y de los aspectos administrativos y contables relacionados con este entorno, este adiestramiento debe incluir el conocimiento de los manuales sobre normas y procedimientos vigentes $\mathrm{o}$ por implementarse en la empresa.

De tal manera, que se debe poner en práctica los lineamientos sugeridos en la propuesta para lograr la disminución de cheques devueltos y actualizarla de acuerdo a sus necesidades y a los requerimientos del entorno.

Del mismo modo, motivar a los integrantes del departamento a proponer nuevas estrategias para garantizar la continuidad de los programas. Las mejoras propuestas se insertan en una visión de la empresa dinámica en la cual el conocimiento y las tecnologías de información y comunicación deben ser utilizadas de manera integrada, coherente $y$ coordinada, soportando la administración y regulación de los recursos financieros, humanos y tecnológicos de la empresa estudiada.

\section{REFERENCIAS BIBLIOGRAFICAS}

Cárdenas, Magdelis, Flores, Yunadys y Parra Greisy, (2011). Control Interno asociado a la Gestión Administrativa Contable de los Inventarios de Materiales y Suministros para la optimización de las operaciones en la Empresa Construcciones y Servicios C.A. Trabajo de Grado presentado en la Universidad de Carabobo

Durán, Carpio, Juan Carlos (2015). El Control Interno de las cuentas por cobrar y su incidencia en la liquidez de la empresa el Mundo Berrezueta Carmona y Cía. Trabajo de grado no publicado. Universidad Técnica de Machala unidad académica de ciencias 
empresariales carrera de Contabilidad y Auditoría. Machala el Oro. Ecuador. Disponible:

http://repositorio.utmachala.edu.ec/bitstrea $\mathrm{m} / 48000 / 4621 / 1 /$ TUACE-2015-CACD00006.pdf. (Consultado: 2016, Octubre 16)

Flores, Nauylud; Rebolledo, Marlene y Sánchez, Odin a (2012).Análisis del ambiente y actividades de control interno del proceso de ventas y cuentas por cobrar basado en las directrices del informe coso en la empresa pastas Sindoni C. A., Ubicada En Maracay, estado Aragua. Trabajo de Grado presentado ante la Universidad de Carabobo para Optar al Título de Licenciado en Contaduría Pública. Bárbula, municipio Naguanagua estado Carabobo

Méndez, Williams (2016). Preparación y análisis de Estados financieros. Recursos instruccionales. Universidad de los Andes facultad de Ciencias Económicas Y Sociales escuela de Administración y Contaduría Pública Mérida. Venezuela Disponible: http://webdelprofesor.ula.ve/economia/me ndezm/. (Consultado: 2017, octubre 14)

Marín María (2014). El control interno basado en el modelo COSO. Documento en línea. Disponible:

revistascientificas.upeu.edu.pe/index.php/ri article/download/334/342. (Consultado: 2017, octubre 14)

Pablos, G; Ramírez, Y. y Román, A. (2012). Puntos comunes de la comunicación organizacional y el control interno. Artículo de la Revista Digital Contribuciones a la Economía, de la Universidad de la Tunas, Cuba. Consulta: Abril 14, 2016. Disponible en:http://www.eumed.net/ce/2010a/sfp.htm

Quintero, Ana (2012). Diseño de estrategias para la conducción de las cuentas por cobrar dirigidas a optimizar la rentabilidad financiera en las empresas inversoras de seguros ubicadas en los Colorados Valencia. Trabajo de grado de maestría no publicado. Universidad de Carabobo. Bárbula Naguanagua. Estado Carabobo 